\title{
Five-year follow-up of an evidence-based prescribing intervention
}

\section{AIMS AND METHOD}

We studied the impact of an evidence-based multidisciplinary intervention to reduce six sub-optimal aspects of psychotropic prescribing, combined as a Prescribing Practice Quality (PPQ) score over a 5-year follow-up period in a community mental health service.

\section{RESULTS}

Sub-optimal prescribing practices were significantly reduced after 1 year and these improvements were sustained at 5-year follow-up. The PPQ scores were significantly reduced $(P<0.001)$ in both the overall population attending at each follow-up point as well as in the everpresent population $(n=163)$. Use of high-dose antipsychotics and thioridazine ceased entirely; use of sedative hypnotic agents was less amenable to reduction.

\section{CLINICAL IMPLICATIONS}

Multifaceted interventions can achieve sustained improvements in prescribing practices in real-world settings.
Despite the drive to standardise clinical practice according to evidence-based guidelines, factors that relate to implementation in routine practice are not well-studied (Bauer, 2002). Dissemination of guidelines and simple educational interventions appear to have limited impact. Practice change is more likely where it involves multifaceted approaches that target various resistance points and are supported by resource allocation, regular reminders and monitoring of practice (Dollman et al, 2005). Moreover, even where change occurs, less is known about sustaining improvements, with evidence indicating that short-term gains are difficult to maintain over time (Caswell et al, 2006).

Although information on the use of psychotropic agents is readily available, prescribing in real-world settings frequently deviates from suggested guidelines and licensing arrangements (Harrington et al, 2002; Hodgson \& Belgamwar, 2006; Spinewine et al, 2007). A recent review by the Royal College of Psychiatrists (2007) highlighted the lack of data regarding the frequency of unlicensed prescribing in UK services, but European studies indicate that up to $66 \%$ of out-patient antipsychotic prescriptions are for unlicensed indications. In part, this reflects the complexities of real-world clinical practice where patient profiles often differ considerably from those of efficacy studies. Combination strategies, for example, benefit many patients with difficult-to-treat illness but remain insufficiently studied and not included within licensed indications (Karow \& Lambert, 2003). However, other practices are discouraged because of concerns over safety such as increased risk of accidents (Department of Health and Children, 2002) or cardiotoxicity (Reilly et al, 2000).

We have previously investigated factors associated with six aspects of psychotropic prescribing (Meagher \& Moran, 2003) and studied the impact of a multidisciplinary intervention on practices at 1-year (Moran et al, 2006) and 2-year (Raju \& Meagher, 2005) followup. This work indicated that sub-optimal prescribing practices (e.g. polypharmacy and use of maintenance benzodiazepines) was substantially reduced by an intervention involving clear guidelines for discontinuation, education, provision of alternative non-pharmacological supports (e.g. anxiety management interventions) and regular monitoring of prescribing patterns. We further studied prescribing practices as part of an annual prescribing audit 5 years after the initial intervention to identify the extent to which improvements in prescribing were sustained at 5-year follow-up, the success in reducing individual sub-optimal practices, and factors associated with sustained improvement.

\section{Method}

The study was conducted in the St Anne's Community Mental Health Service, which provides a general adult psychiatry service for a catchment area of approximately 50000 persons in south-east Limerick. We documented six aspects of sub-optimal psychotropic prescribing rated as present (1) or absent (0) to produce a Prescribing Practice Quality (PPQ) score (Box 1), with higher scores reflecting less optimal practice. Previous work identifying determinants of sub-optimal prescribing and the impact of a multidisciplinary intervention on practice has described the PPQ (including item definitions) and the multicomponent intervention in detail (Meagher \& Moran, 2003; Raju \& Meagher, 2005; Moran et al, 2006).

In the period since the original intervention there has been continued emphasis on the agreed prescribing protocols, with a brief educational session for each new doctor as part of service induction. In addition, an annual audit allows monitoring of service contacts, ICD-10 diagnoses and prescribing practices within the service, and allows feedback as to prescribing patterns as part of ongoing service review. Two specific aspects of practice were not considered 'sub-optimal' for the purposes of this study because they reflected specific agreed practices in the service: the first was targeted polypharmacy with the combination of a serotonin-noradrenaline reuptake inhibitor (either venlafaxine or duloxetine) with mirtazapine for treatment-resistant depressive illness (Meagher et al, 2006; Hannon et al, 2007), and the 


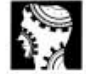

original papers
Box 1. Prescribing Practice Quality (PPQ) items and scoring

- Polypharmacy (i.e. use of two agents of same class)

- Receiving thioridazine

- High-dose antipsychotic use (total dose greater than $1000 \mathrm{mg} /$ day in chlorpromazine equivalents)

- Maintenance benzodiazepine treatment

- Maintenance hypnotic treatment

- Routine use of anticholinergic agent

Scoring: 1 point per item present; range $0-6$; higher scores indicate less optimal prescribing.

second was short-term use of quetiapine in low doses (25-100 mg) as a hypnotic agent.

Statistical analysis was conducted using SPSS version 14 for Windows. The PPQ scores for total populations at each point were compared with Wilcoxon signed ranks testing. Independent $t$-tests were used to compare age and duration of service attendance. Chi-squared tests were used to compare the frequency of severe mental illness, depot antipsychotic treatment and individual PPQ items between those ever-present in the service and those not.

\section{Results}

Table 1 shows the demographic and clinical characteristics of service attenders at initiation of the programme (in 2001), at 1-year follow-up (2002) and at 5-year follow-up (2006). The overall case-load, age, gender balance, duration of service attendance and percentage with severe mental illness were similar over the 5 years. Table 2 shows the frequency of sub-optimal prescribing practices (both

Table 1. Demographic and clinical characteristics (2001-2006)

\begin{tabular}{lccc} 
& 2001 & 2002 & 2006 \\
\hline $\begin{array}{l}\text { Service attenders, } n \\
\text { Age, years: mean (s.d.) }\end{array}$ & $405(14)$ & $43(15)$ & $44(14)$ \\
$\begin{array}{l}\text { Male, \% } \\
\text { Duration of service } \\
\text { attendance, years: }\end{array}$ & 49 & 51 & 47 \\
$\begin{array}{l}\text { mean (s.d.) } \\
\text { Patients with severe } \\
\text { mental illness, \% }\end{array}$ & $6.8(7.7)$ & $7.5(8.4)$ & $6.2(7.4)$ \\
\hline
\end{tabular}

overall and individual items) at the three time points. There was a consistent reduction in mean PPQ score for the total population at each assessment point as well as a reduction in the percentage of patients with at least one sub-optimal aspect of their prescribed medications, from almost half in 2001 (46\%) to just over a quarter in 2006 (27\%).

Two aspects of prescribing - use of thioridazine, and high-dose antipsychotic treatment - ceased entirely over the course of the study. In contrast, the use of anticholinergic agents and benzodiazepines declined during the initial phase of the study (2001-2) but remained relatively stable between 2002 and 2006, indicating that the initial reduction in use was sustained over the longer period of follow-up. The specific hypnotic agents prescribed did alter, with relatively greater use of zopiclone over time: $n=24$ ( $23 \%$ of total hypnotic use) in 2001, $n=24(30 \%)$ in 2002 and $n=30(49 \%)$ in 2006.

A total of 163 patients were clients of the service at all three evaluation points. These 'ever-present' patients were older than the general population attending in 2006 $(P<0.001)$, and were more likely to have severe mental illness $(66 \%$ v. $26 \%, P<0.001)$ and to be in receipt of depot antipsychotic treatment $(18 \%$ v. $3 \%, P<0.001)$. The mean PPQ scores for these patients are shown in Table 3. The percentage of patients from this population who had at least one aspect of prescribing that was sub-optimal (i.e. a PPQ score of 1 or more) diminished over time from $46 \%$ in 2001 to $38 \%$ in 2002 and $33 \%$ in 2006. Mean PPQ scores were significantly reduced at 2002 v. 2001 $(P<0.001)$ and at $2006 \mathrm{v}$. both $2001(P<0.001)$ and $2002(P<0.001)$.

The ever-present population was further subdivided into those who had maintained improved prescribing $(n=58)$ and those who had not $(n=105)$ at 5 -year followup. A greater likelihood of being in the improved group was associated with higher baseline PPQ score: 0.2 (s.d. $=0.5)$ v. 2.1 (s.d. $=0.9$ ), $P<0.001$. There were trends towards greater likelihood of having improved PPQ scores in those with severe mental illness $(P=0.09)$ and older age $(P=0.06)$. Improved PPQ score was not related to gender, duration of service attendance, treatment with depot antipsychotic medication, frequency of attendance at services within the previous year or receipt of multidisciplinary shared care (i.e. attending at least two members of the multidisciplinary team).

Table 2. Sub-optimal prescribing practices (2001-2006)

\begin{tabular}{|c|c|c|c|}
\hline & 2001 & 2002 & 2006 \\
\hline Patients with at least one sub-optimal prescribing practice, $\%$ & 46 & 38 & 27 \\
\hline \multicolumn{4}{|l|}{ Individual aspects of prescribing } \\
\hline High-dose antipsychotic use & 10 & 6 & 0 \\
\hline Polypharmacy & 60 & 30 & 19 \\
\hline Thioridazine use & 36 & 4 & 0 \\
\hline Maintenance benzodiazepine & 78 & 41 & 49 \\
\hline Maintenance use of sedative/hypnotic & 106 & 57 & 61 \\
\hline
\end{tabular}

$P P Q$, Prescribing Practice Quality. 
Table 3. Prescribing Practice Quality scores for 'ever-present' patients $(n=163)$

\begin{tabular}{|lccc}
\hline & 2001 & 2002 & 2006 \\
\hline $\begin{array}{l}\text { PPQ score: mean } \\
\text { (s.d.) }\end{array}$ & $0.86(1.1)$ & $0.64(0.98)$ & $0.39(0.60)$ \\
\hline
\end{tabular}

$P P Q$, Prescribing Practice Quality.

Thirty-nine patients were receiving depot antipsychotic agents at the 2006 review. These patients were significantly older (mean age 55.9 years, s.d. $=12.4$; $P<0.001$ ), had attended the service for longer (mean 15.9 years, s.d. $=10.3 ; P<0.001)$ and had significantly higher PPQ scores (mean score 0.85 , s.d. $=0.78$;

$P<0.001)$ than those not receiving depot antipsychotic medication. This reflected a high rate of anticholinergic use (52\%) and concomitant oral antipsychotic use (38\%) in this population.

\section{Discussion}

We report a 5-year follow-up of an evidence-based prescribing intervention in a real-world setting indicating that improvements in prescribing quality can be maintained over time. Improvements were evident both in the ongoing 'ever-present' population as well as the total population attending the service at follow-up, indicating a reduction in sub-optimal practices in both pre-existing cases and where new medication was initiated. These benefits were maintained by minimal formal intervention - an initial education session incorporated into the induction process focusing on policies around suboptimal practices supported by emphasis of alternative strategies in day-to-day practice (e.g. multidisciplinary team meetings) and regular audit with feedback at an annual service review. Our study indicates that change driven by the collaborative efforts of clinical staff, and therefore sensitive to the demands of everyday practice within that service (including local resource strengths and limitations), produced sustained improvement in practice. Coercive approaches (e.g. mandatory prescribing restrictions) are effective in changing practice (Wagner et al, 2007) but can be associated with increased subsequent polypharmacy, reduced treatment adherence and greater in-patient and emergency service utilisation (Tamblyn et al, 2001; Parks \& Surles, 2004; Soumerai, 2004). We did not find any increase in polypharmacy, and admission rates decreased over the study period, suggesting that in-patient service use and non-compliance-related relapses were not a common consequence. The specific approach to benzodiazepine use in our programme involved patient-controlled dose reduction (Raju \& Meagher, 2005) emphasising the advantages of collaboration between patients and clinical staff in delivering practice change.

Measuring the longer-term impact of interventions and identifying predictors of change is complicated by factors illustrated in this study. The only identified positive predictor of improved prescribing practice at 5-year follow-up was baseline PPQ score. We previously identified exposure to adjunctive supportive inputs (e.g. anxiety management) and chronicity of sub-optimal prescribing practice as predictors of improved prescribing at 1-year follow-up, but these factors were difficult to measure over more prolonged periods. It is interesting that patients receiving depot antipsychotic medications continued to have less than optimal prescribing despite being previously highlighted for particular attention (Meagher \& Moran, 2003), reflecting the tendency for use of anticholinergic agents and antipsychotic polypharmacy in this group. In addition, the PPQ was affected by changing practices, with some of the original measures (e.g. use of thioridazine) now extremely uncommon and less useful indicators of prescribing quality. Moreover, even though some aspects of prescribing are enduring indicators (polypharmacy, benzodiazepine use), the specific agents involved changed over time.

Where polypharmacy is driven by emerging evidence of efficacy, or as a means to reduce side-effects (e.g. clozapine augmentation with atypical antipsychotics), it can reflect optimal practice. Moreover, polypharmacy may be driven by demonstrated benefits for individual patients even if efficacy studies are lacking. It may be that optimal patient outcomes require a low frequency of practices that are generally considered sub-optimal but have demonstrable benefits for individual patients. Moreover, clinical practice is sometimes more advanced than standards identified by randomised controlled trials or that use specifically licensed by regulatory bodies. However, it is often the case that in reality much suboptimal prescribing reflects educational deficits or other constraints such as a lack of access to alternate interventions for disabling problems (Stone et al, 2002) and often occurs by default rather than design. This spectrum of prescribing is highlighted in a recent report (Royal College of Psychiatrists, 2007) and ranges from 'near-label' prescribing to practices that have little foundation in evidence.

Benzodiazepine use is associated with risk of dependence, daytime sedation, cognitive impairment and falls (American Psychiatric Association, 1990). Although use of this class of drugs is discouraged in prescribing guidelines, it is common in real-world settings and is often resistant to change (Oude Voshaar et al, 2003; Caswell et al, 2006). It is interesting that reduction in use does not necessarily equate with lower subsequent incidence of associated adverse events such as falls (Wagner et al, 2007), emphasising the gap that can exist between clinical activities and presumed benefits in patient outcomes. In our population we noted a gradual increase in use of zopiclone, in keeping with a perception of better tolerability and reduced dependency potential compared with benzodiazepines, but gathering evidence of misuse and dependence potential needs to be noted (Mahomed et al, 2002). Other work suggests low-dose antipsychotics as a relatively benign substitute for benzodiazepines (Hamann et al, 2003), but emerging evidence of metabolic and cerebrovascular effects is a cause for considerable concern (Lieberman, 2004; Schneider et al, original papers 


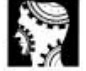

original papers
2005) and emphasises the need for careful monitoring where these agents are used 'off licence'.

Some aspects of prescribing are more difficult to reduce owing to factors such as patient reluctance (Oude Voshaar et al, 2003; Raju \& Meagher, 2005) as well as perceived clinical benefit. Some have justified long-term benzodiazepine treatment for patients with disabling and treatment-refractory anxiety disorders where benzodiazepine use is associated with enhanced quality of life (Williams \& McBride, 1999), but the characteristics of these patients remain unclear and evidence points to over- rather than under-utilisation in current practice. In the case of anticholinergic use, current opinion (World Health Organization, 1990) suggests that approximately $5 \%$ of patients receiving typical antipsychotic treatment require maintenance anticholinergic treatment. Consequently, a low level of use is consistent with optimal management of extrapyramidal symptoms. It remains unclear to what extent further reduction in these practices is consistent with optimal outcomes for patients. The need for evidence linking greater guideline adherence with improved patient outcomes is highlighted by Simpson et al (2005), who linked strict enforcement of National Institute for Health and Clinical Excellence (NICE) guidelines around cholinesterase inhibitor use to poorer patient outcomes. Other work (e.g. Mortimer et al, 2005), using similar parameters of sub-optimal prescribing to those used in our study, has linked greater side-effect burden to less-optimal prescribing that reduced as these practices diminished.

Ultimately a balanced approach to managing prescribing is required. Although some practices are clearly undesirable, others are acceptable for a minority of patients. The desire for greater evidence-based practice needs to recognise the limited capacity of available knowledge to guide many aspects of treatment (especially augmentation and combination therapy) as well as respecting the autonomy and judgement of clinicians and patients in identifying optimal individualised therapeutic plans. As a basic principle, where prescribing deviates from suggested norms, this should reflect targeted pharmacological management, the benefits of which are carefully monitored over time.

\section{Declaration of interest}

D.M. has received an unrestricted educational grant from AstraZeneca Pharmaceuticals.

\section{References}

AMERICAN PSYCHIATRIC ASSOCIATION (1990) Benzodiazepine Dependence, Toxicity, and Abuse: A Task Force Report. American Psychiatric Association.

BAUER, M. S. (2002) A review of quantitative studies of adherence to mental health clinical practice guideline. Harvard Review of Psychiatry, 10, 138-153.

CASWELL, L., HOOSEN, I., VASSILAS, C. A., et al (2006) Reducing hypnotic use in two older adult functional wards: (2002) Report of the Benzodiazepine Committee. Republic of Ireland Government Publications.

DOLLMAN, W. B., LEBLANC, V.T. STEVENS, L., et al (2005) Achieving a sustained reduction in benzodiazepine use through implementation of an areawide multistrategic approach. Journal of Clinical Pharmacy and Therapeutics, 30, 425-432 Pharmaceutical Journal, 268, 657-659.
HAMANN, J., RUPPERT, A., AUBY, P., et al (2003) Antipsychotic prescribing patterns in Germany: a retrospective analysis using a large outpatient prescription database. International Clinical Psychopharmacology, 18, 237-242

HANNON, N., HAMZAH, Z AKINPELOYE, H., et al (2007) Venlafaxine-mirtazapine combination in the treatment of persistent depressive illness. Journal of Psychopharmacology, 21, 161-165.

HARRINGTON, M., LELLIOTT, P., PATON, C. et al (2002) The results of a multicentre audit of the prescribing of antipsychotic drugs for in-patients in the UK. Psychiatric Bulletin, 26, 414-418.

HODGSON, R. \& BELGAMWAR, R. (2006) Off-label prescribing by psychiatrists. Psychiatric Bulletin, 30 , 55-57.

KAROW, A. \& LAMBERT, M. (2003) Polypharmacy in treatment with psychotropic drugs: the underestimated phenomenon. Current Opinion in Psychiatry, 16, 713-718.

LIEBERMAN, J. A. (2004) Metabolic changes associated with antipsychotic use. Journal of Clinical Psychiatry, 6 (suppl 2), 8-13.

MAHOMED, R., PATON, C. \& LEE, E. (2002) Prescribing hypnotics in a mental health trust: what consultant psychiatrists say and what they do.

MEAGHER, D. \& MORAN, M. (2003) Sub-optimal prescribing in an adult community mental health service: prevalence and determinants. Psychiatric Bulletin, 27, 266-270.

MEAGHER, D., HANNAN, N. \& LEONARD, M. (2006) Duloxetinemirtazapine combination in depressive illness: the case for Limerick 'rocket fuel'. Irish Journal of Psychological Medicine, 23,116-118.

MORAN, M., RAJU, B. \& SAUNDERS, J. et al (2006). Achieving evidence-based prescribing practice in an adult community mental health service. Psychiatric Bulletin, 30, 51-55.

MORTIMER, A. M., SHEPHERD, C. J. RYMER, M., et al (2005) Primary care use of antipsychotic drugs: an audit and intervention study. Annals of General Psychiatry, 4, 18.

PARKS, J. \& SURLES, R. (2004) Using best practices to manage psychiatric medications under Medicaid. Psychiatric Services, 55, 1227-1229.

RAJU, B. \& MEAGHER, D. (2005) Patient-controlled benzodiazepine dose reduction in a community mental

health service. Irish Journal of Psychological Medicine, 22, 42-45.

REILLY, J. G., AVIS, S. A., FERRIER, I. N., et al (2000) QTc-interval abnormalities and psychotropic drug therapy in psychiatric patients. Lancet, 355, 1048-1052.

ROYAL COLLEGE OF PSYCHIATRISTS (2007) Use of Licensed Medicines for Unlicensed Applications in Psychiatric Practice (College Report CR142). Royal College of Psychiatrists.

SCHNEIDER, L. S., DAGERMAN, K. S. \& INSEL, P. (2005) Risk of death with atypical antipsychotic drug treatment for dementia: meta-analysis of randomized placebo-controlled trials. JAMA, 294, 1934-1943.

SIMPSON, S., BEAVIS, D., LEDDY, A., et al (2005) Naturalistic audit of NICE criteria for the use of cholinesterase inhibitors. Psychiatric Bulletin, 29 $410-412$

SOUMERAI, S. B. (2004) Benefits and risks of increasing restrictions on access to costly drugs in Medicaid. Health Affairs, 23, 135-146.

SPINEWINE, A., SWINE, C., DHILLON, S., et al (2007) Effect of a collaborative approach on the quality of prescribing for geriatric inpatients: a randomised controlled trial. Journal of the American Geriatrics Society, 55, 658-665.

STONE, J., OHISEN, R., TAYLOR, D , et al (2002) Naturalistic study of the antipsychotic medication review service at the Maudsley Hospital. Psychiatric Bulletin, 26, 291-294.

TAMBLYN, R., LAPRISE, R., HANLEY, J. A., et al (2001) Adverse events associated with prescription drug costsharing among poor and elderly persons. JAMA, 285, 421-429.

VOSHAAR, R. C., GORGELS,W. J. MOL, A. J., et al (2003) Tapering off long-term benzodiazepine use with or without group cognitive-behavioural therapy: three-condition, randomised controlled trial. British Journal of Psychiatry, 182, 498-504.

WAGNER, A. K., ROSS-DEGNAN, D., GURWITZ, J. H., et al (2007) Effect of NewYork state regulatory action on benzodiazepine prescribing and hip fracture rates. Annals of Internal

Medicine, 146, 96-103.

WILLIAMS, D. D. R. \& MCBRIDE, A (1999) Benzodiazepines: time for reassessment. British Journal of Psychiatry, 173, 361-362.

WORLD HEALTH ORGANIZATION (1990) Prophylactic use of anticholinergics in patients on long-term neuroleptic treatment. A consensus statement. British Journal of Psychiatry, 156, 412.

David Meagher Professor, Department of Health Services Research, University of Limerick, and Department of Adult Psychiatry, Midwestern Regional Hospital, Limerick, Republic of Ireland, email: davidjmeagher@gmail.com, Ananth Pullela Consultant Psychiatrist, Midwestern Regional Hospital, Limerick,

Marek Meisinger Registrar in Psychiatry, Niamh Geaney Senior House Officer, Sinead O'Brien Senior Registrar, Limerick Mental Health Services, Limerick, Republic of Ireland 